Research Paper

\title{
The Degree of Lipiodol Accumulation Can Be an Indicator of Successful Treatment for Unresectable Hepatocellular Carcinoma (HCC) Patients - in the Case of Transcatheter Arterial Chemoembolization (TACE) and External Beam Radiotherapy (EBRT)
}

\author{
Ping Yang, ${ }^{1}$ Zhao-Chong Zeng, ${ }^{1 凶}$ Bin-Liang Wang, ${ }^{1}$ Jian-Ying Zhang, ${ }^{1}$ Jia Fan, ${ }^{2}$ Jian Zhou, ${ }^{2}$ Yong Hu${ }^{1}$ \\ 1. Department of Radiation Oncology, Zhongshan Hospital, Fudan University, Shanghai 200032, China. \\ 2. Department of Liver Cancer Institute, Zhongshan Hospital, Fudan University, Shanghai 200032, China. \\ $\triangle$ Corresponding author: Zhao-Chong Zeng, MD. Department of Radiation Oncology, Zhongshan Hospital, Fudan University, 180 Feng Lin Road, Shanghai \\ 200032, China. Tel: 86-21-64041990; Fax: 86-21-64038472 E-mail: zeng.zhaochong@zs-hospital.sh.cn.
}

( ) Ivyspring International Publisher. Reproduction is permitted for personal, noncommercial use, provided that the article is in whole, unmodified, and properly cited. See http://ivyspring.com/terms for terms and conditions.

Received: 2016.02.29; Accepted: 2016.05.18; Published: 2016.07.04

\begin{abstract}
Purpose: Transcatheter arterial chemoembolization (TACE) in combination with external beam radiotherapy (EBRT) results in improved survival due to better local control in patients with unresectable hepatocellular carcinoma (HCC). The purpose of this study was to investigate lipiodol accumulation, as it reflects tumor burden and is a potential prognostic factor, in $\mathrm{HCC}$ patients treated with TACE/EBRT.

Methods and Materials: We retrospectively studied 147 patients with unresectable HCC treated with TACE and EBRT. Clinical features, adverse reactions, and prognostic factors were analyzed. All patients were treated with TACE 1-6 times in combination with EBRT (44-66 Gy) in dose of 2 Gy/fraction given once a day five times a week. Tumor status and laboratory findings were followed. The degree of lipiodol accumulation was assessed by computed tomography before EBRT, and was categorized as either complete/intense or low/moderate.

Results: The response rate of tumor size after EBRT was 68.2\%, median survival was 23.1 months, and overall survival rates were $86.6 \%, 49.2 \%$, and $28.2 \%$ at 1,2 , and 3 years, respectively. Univariate analysis showed that lower hemoglobin levels, higher alkaline phosphatase levels, Child-Pugh B, negative alpha-fetoprotein (AFP) response after EBRT, poor treatment response after EBRT, tumor diameter $>10 \mathrm{~cm}$, and poor lipiodol accumulation were unfavorable prognostic factors. On multivariate analysis, higher hemoglobin levels, Child-Pugh A, decreased AFP levels after treatment, Helical Tomotherapy $(\mathrm{HT})$ and intense lipiodol accumulation after TACE were significant favorable predictors.

Conclusions: The degree of lipiodol accumulation before EBRT is a prognostic factor in patients with unresectable HCC. Increased AFP levels after EBRT are always associated with poor survival. HT is recommended as a potentially better EBRT modality than three-dimensional conformal radiation therapy (3D-CRT).
\end{abstract}

Key words: External beam radiation therapy (EBRT); transcatheter arterial chemoembolization (TACE); hepatocellular carcinoma (HCC); lipiodol accumulation.

\section{Introduction}

Hepatocellular cancer (HCC) is one of the most common malignancies, currently resulting in 466,100 new cases and 422,100 deaths per year in China [1].
Hepatic resection or liver transplantation is the first choice of treatment, but these options are rarely possible because of associated liver disease, tumor 
multiplicity, and/or extension. It remains challenging to treat patients with unresectable HCC, treatment modalities like percutaneous ethanol injection (PEI) and radiofrequency ablation (RFA) are effective but seem to be more suitable in smaller tumors [2]. For patients with large tumors, complete or massive necrosis is seldom observed when treated with Transcatheter arterial chemoembolization (TACE) alone. External beam radiation therapy (EBRT) has often been used in conjunction with TACE and better responses have been reported [3], possibly due to the relief in tumor burden after TACE. However, there have been few reports on whether the tumor burden affects its response to radiation. The purpose of this study was to examine prognostic factors in detail, especially those that reflect tumor burden in unresectable HCC patients before EBRT.

\section{Methods and Materials}

\section{Patients and diagnosis}

From January 2004 to December 2014, 147 patients with unresectable HCC received combined TACE and EBRT treatment at Zhongshan Hospital, Fudan University and were investigated retrospectively. Diagnosis of HCC was confirmed by histologic testing (biopsy or surgical specimen) in 35 patients $(23.8 \%)$, or the typical radiologic features in a cirrhotic liver with or without an elevated serum AFP level in 112 patients (76.2\%). Typical images to diagnose the presence of HCC in a mass lesion greater than $1 \mathrm{~cm}$ in greatest dimension with hypervascularity were taken [4]. Unresectable tumor was defined by extensive tumor, major vascular invasion, or a poor medical condition that disallows surgical resection. After HCC was diagnosed, a Child-Pugh test was scored based on levels of serum bilirubin and serum albumin, prothrombin time prolongation, presence or absence of ascites, and encephalopathy. Patients with Karnofsky performance status (KPS) of $<80$, hepatic function by Child-Pugh classification C, vascular tumor thrombus, lymph node involvement, or distant metastasis were excluded.

\section{Therapies}

TACE was offered to unresectable HCC patients as an initial therapy and was performed in 1-6 separate treatments (median of 3 treatments) at $\geq 4$-week intervals, using a combination of targeted chemotherapy with $1 \mathrm{~g}$ 5-fluorouracil (5-FU), $80 \mathrm{mg}$ cisplatin (DDP), $10 \mathrm{mg}$ mitomycin $\mathrm{C}(\mathrm{MMC})$, and arterial embolization with 5-30 mL (median of $10 \mathrm{~mL}$ ) iodized oil (Lipiodol ultra fluid) mixed with $10 \mathrm{mg}$ MMC.
EBRT was delivered using three-dimensional conformal radiation therapy (3D-CRT) or helical tomotherapy (HT). CT simulation of the abdomen was used for designs and delivery of EBRT. All patients were immobilized with a posterior vacuum fixation device, as for HT patients, an anterior pressure plate was added to reduce respiration movement. The gross tumor volume (GTV) was delineated according to contrast-enhancing tumor exhibiting on diagnostic CT or magnetic resonance imaging (MRI) images. The whole intrahepatic tumor was included in the GTV with physician's concern about tumor size, preserved liver function, and the irradiated volume of the liver. A margin of 5 to $10 \mathrm{~mm}$ was added to the GTV to form clinical tumor volume (PTV). Another 7 to $10 \mathrm{~mm}$ radical margin and 10 to $15 \mathrm{~mm}$ craniocaudal margin were added to count for organ motion and setup errors (planning target volume (PTV). The normal liver volume was defined as the entire liver volume minus the GTV. Treatment planning was generated using $\mathrm{XiO}$ treatment planning system in 3D-CRT patients and Tomotherapy planning software, version 4.2 (Tomotherapy, Madison, WI) in HT patients. The median total dose was 50 Gy (range of 40-66 Gy) at 2 Gy/fraction, five times a week (once per day). Patients with large tumors, with less normal tissue reserve, or with severe liver cirrhosis should be delivered lower dose. However, a higher dose of radiation therapy is effective for small tumors, with frank sparing of non-tumorous adjacent liver parenchyma. Choice of treatment doses is based individually on the dose of organs at risk.

The organs at risk used in current study were 1) spinal cord: maximum dose $\leq 45 \mathrm{~Gy} ; 2$ ) kidneys: mean dose to bilateral kidneys must be $<15 \mathrm{~Gy}$, and no more than $50 \%$ of the volume of kidney can receive $\geq 20$ Gy; 3) liver: mean normal liver dose must be $\leq 30 \mathrm{~Gy}$ (3D-CRT) or $\leq 28$ Gy (HT), and no more than $60 \%$ of normal liver can receive $\geq 30$ Gy (V30<60\%); and 4) stomach and small bowels: maximum dose $\leq 55 \mathrm{~Gy}$, and no more than $5 \%$ of small bowels can receive $\geq 50$ Gy $(\mathrm{V} 50<5 \%)$.

Both written and oral informed consent was obtained from each patient in a standard procedure at each department. The procedures used were in accordance with the ethical standards of the responsible committee on human experimentation (institutional or regional) and with the Helsinki Declaration of 1975, as revised in 2004.

\section{Assessment of response and toxicity}

Pretreatment evaluation included a medical history and physical examination, complete blood cell counts, serum chemistries, liver function tests, AFP 
test, chest X-ray, abdominal ultrasonography, enhanced CT or MRI (or both), and hepatic angiography. We assessed the tumor burden before EBRT by four parameters: tumor size (diameter in $\mathrm{cm}$ ), number of intrahepatic tumors (single or multiple), initial AFP level $(<20 \mu \mathrm{g} / \mathrm{L}, 20-400 \mu \mathrm{g} / \mathrm{L}$, or $\geq 400 \mu \mathrm{g} / \mathrm{L}$ ), and lipiodol accumulation after TACE.

Traditionally, lipiodol accumulation patterns are classified into four types (type I, homogeneous; type II, defective; type III, inhomogeneous; and type IV, only slight accumulation)[5]. We used a simpler scheme of two types: type I, complete/intense accumulation and type II, low/moderate accumulation (Figure 1). We assessed lipiodol accumulation by CT before EBRT (4 weeks after the last TACE). We delineated the intrahepatic tumors and intratumoral lipiodol by non-contrast CT, measured the tumor volume ( $\left.\mathrm{V}_{\text {tumor }}\right)$ and the lipiodol volume ( $\left.\mathrm{V}_{\text {lipiodol }}\right)$, and then calculated the ratio of $\mathrm{V}_{\text {lipiodol }} / \mathrm{V}_{\text {tumor }}$. We categorized ratios $>3 / 4$ as complete/intense lipiodol accumulation and those $\leq 3 / 4$ as low/moderate lipiodol accumulation.

Patients were monitored with abdominal enhanced CT or MRI approximately 1.5 months after completion of EBRT (first follow-up) and every 3 months thereafter. The response evaluation to criteria in solid tumors (RECIST) was used to determine the tumor response after irradiation [6]. Complete response (CR) represented complete disappearance of the irradiated tumor. Partial response (PR) represented at least a $30 \%$ reduction of tumor in the greatest dimension. Progressive disease (PD) was defined as at least a $20 \%$ increase of tumor in the greatest dimension, and stable disease (SD) was defined as any tumor volume change other than PR or $\mathrm{PD}$. Objective response was calculated for CR and PR; no response was calculated for SD or PD.

The overall survival (OS) period was defined as the period from the date of the first TACE treatment to the date of death or the last follow-up appointment.

\section{Statistical analysis}

Cumulative survival rates were analyzed by Kaplan-Meier plots, and differences between the curves were assessed by the log-rank test. For multivariate analysis, all variables were entered using the backward Wald method. $\mathrm{P}<0.05$ was considered statistically significant. All calculations were performed with SPSS 19.0 software for Windows (SPSS, Chicago, IL, USA).

\section{Results}

\section{Patients' characteristics}

The cohort included 117 men and 30 women with a median age of 56 years (range, 28-85 years). Pretreatment variables that showed significant differences in survival in the univariate analysis, including demographics, clinical laboratory tests, tumor status, and treatment information for all patients, are listed in Table 1.
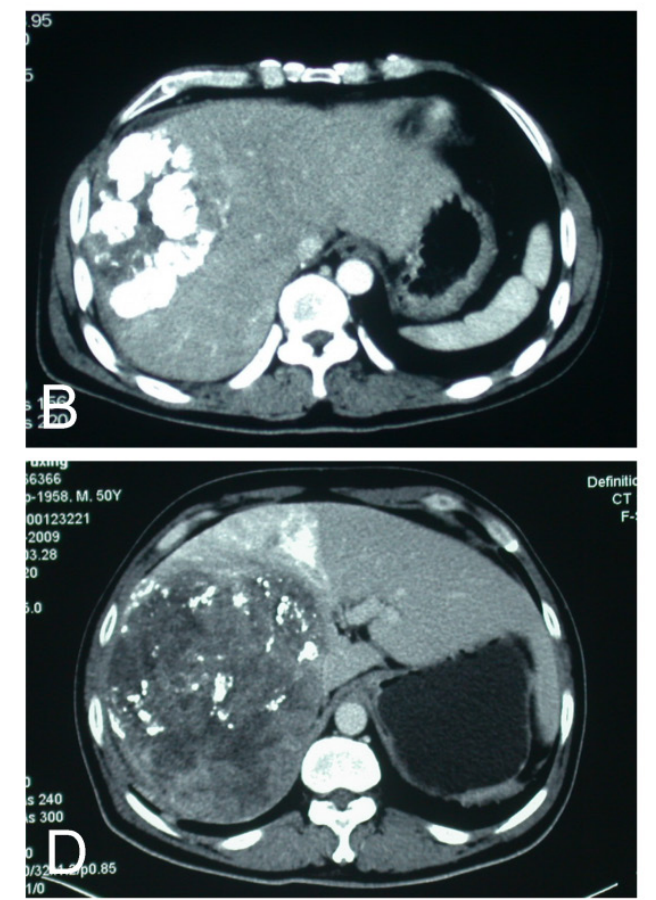

Figure 1. CT scans of patterns of lipiodol accumulation after TACE. (a) Complete; (b) intense (>75\% of tumor volume); (c) moderate ( $\leq 75 \%$ of tumor volume; (d) low. 
Table 1. Univariate and multivariate analysis of baseline predictors of survival in 147 patients with unresectable hepatocellular carcinoma.

\begin{tabular}{|c|c|c|c|c|c|c|c|c|}
\hline \multirow[t]{3}{*}{ Variables } & & \multirow[t]{3}{*}{$\mathrm{n}$} & \multicolumn{2}{|c|}{ Survival status } & \multicolumn{3}{|c|}{$\mathrm{P}$} & \multirow{3}{*}{$\begin{array}{c}\text { Correlation with } \\
\text { LA } \\
(\mathrm{P})\end{array}$} \\
\hline & & & \multirow[t]{2}{*}{ Average $\pm S D$} & \multirow[t]{2}{*}{ Median \pm SD } & \multirow[t]{2}{*}{ Univariate } & \multicolumn{2}{|c|}{ Multivariate } & \\
\hline & & & & & & With LA & w/o LA & \\
\hline \multirow{3}{*}{$\begin{array}{l}\text { Age } \\
\text { (years) }\end{array}$} & $<40$ & 21 & $28.4 \pm 7.2$ & $18.5 \pm 8.0$ & 0.519 & 0.555 & 0.328 & 0.072 \\
\hline & $40-60$ & 69 & $36.7 \pm 4.3$ & $28.7 \pm 4.9$ & & & & \\
\hline & $\geq 60$ & 57 & $35.8 \pm 4.1$ & $31.5 \pm 6.9$ & & & & \\
\hline \multirow[t]{2}{*}{ Gender } & Female & 30 & $32.8 \pm 2.8$ & $26.3 \pm 5.6$ & 0.887 & 0.750 & 0.456 & 0.907 \\
\hline & male & 117 & $35.2 \pm 3.1$ & $26.6 \pm 3.8$ & & & & \\
\hline \multirow[t]{3}{*}{ KPS } & 100 & 72 & $34.3 \pm 3.7$ & $26.3 \pm 2.2$ & 0.997 & 0.587 & 0.639 & 0.082 \\
\hline & 90 & 63 & $36.7 \pm 5.2$ & $23.8 \pm 6.0$ & & & & \\
\hline & 80 & 12 & $27.9 \pm 4.3$ & $32.9 \pm 11.9$ & & & & \\
\hline \multirow{3}{*}{$\begin{array}{l}\text { Hemoglobin } \\
(\mathrm{g} / \mathrm{L})\end{array}$} & $<110$ & 22 & $19.1 \pm 3.6$ & $12.6 \pm 3.7$ & 0.005 & 0.016 & 0.003 & 0.022 \\
\hline & $110-140$ & 86 & $34.8 \pm 3.5$ & $26.9 \pm 4.4$ & & & & \\
\hline & $\geq 140$ & 39 & $42.6 \pm 6.2$ & $39.7 \pm 7.2$ & & & & \\
\hline \multirow{3}{*}{$\begin{array}{l}\text { Platelet count } \\
(\times 109 / \mathrm{L})\end{array}$} & $<100$ & 54 & $34.4 \pm 4.1$ & $26.3 \pm 5.7$ & 0.306 & 0.802 & 0.976 & 0.511 \\
\hline & $100-200$ & 55 & $41.5 \pm 5.6$ & $31.5 \pm 2.7$ & & & & \\
\hline & $\geq 200$ & 38 & $26.1 \pm 3.3$ & $26.0 \pm 7.0$ & & & & \\
\hline \multirow{2}{*}{$\begin{array}{l}\text { ALP } \\
(\mathrm{IU} / \mathrm{mL})\end{array}$} & $<150$ & 98 & $38.2 \pm 3.3$ & $31.5 \pm 3.0$ & 0.008 & 0.158 & 0.007 & 0.012 \\
\hline & $\geq 150$ & 49 & $21.8 \pm 2.5$ & $16.8 \pm 1.0$ & & & & \\
\hline \multirow{3}{*}{$\begin{array}{l}\text { Y-GT } \\
(\mathrm{IU} / \mathrm{L})\end{array}$} & $<75$ & 53 & $41.3 \pm 4.9$ & $36.3 \pm 6.4$ & 0.116 & 0.176 & 0.061 & 0.284 \\
\hline & $75-150$ & 46 & $35.4 \pm 5.8$ & $22.4 \pm 4.7$ & & & & \\
\hline & $\geq 150$ & 48 & $27.9 \pm 3.9$ & $22.9 \pm 6.0$ & & & & \\
\hline \multirow[t]{2}{*}{ Child-Pugh classification } & A & 94 & $37.3 \pm 3.1$ & $31.5 \pm 3.8$ & 0.005 & 0.037 & 0.017 & 0.040 \\
\hline & B & 53 & $19.4 \pm 3.8$ & $12.1 \pm 1.7$ & & & & \\
\hline \multirow{3}{*}{$\begin{array}{l}\text { AFP status } \\
(\mu \mathrm{g} / \mathrm{L})\end{array}$} & $<20$ & 34 & $39.9 \pm 5.7$ & $31.5 \pm 10.6$ & 0.007 & 0.065 & 0.016 & 0.445 \\
\hline & $20-400$ & 44 & $32.8 \pm 5.4$ & $23.8 \pm 4.6$ & & & & \\
\hline & $\geq 400$ & 69 & $34.2 \pm 4.2$ & $26.9 \pm 5.6$ & & & & \\
\hline \multirow[t]{2}{*}{ AFP response } & positive & 67 & $42.4 \pm 4.2$ & $31.9 \pm 1.0$ & $<0.001$ & $<0.001$ & 0.002 & 0.048 \\
\hline & negative & 46 & $20.4 \pm 2.5$ & $13.8 \pm 2.6$ & & & & \\
\hline \multirow{2}{*}{$\begin{array}{l}\text { Tumor Size } \\
(\mathrm{cm})\end{array}$} & $<10$ & 80 & $43.5 \pm 4.0$ & $36.3 \pm 3.9$ & $<0.001$ & 0.177 & 0.027 & 0.026 \\
\hline & $\geq 10$ & 67 & $21.8 \pm 1.8$ & $18.5 \pm 1.8$ & & & & \\
\hline \multirow{2}{*}{$\begin{array}{l}\text { Intrahepatic tumor } \\
\text { number }\end{array}$} & Single & 109 & $36.5 \pm 3.6$ & $26.6 \pm 3.8$ & 0.438 & 0.547 & 0.969 & 0.784 \\
\hline & multiple & 48 & $32.5 \pm 4.8$ & $23.1 \pm 5.7$ & & & & \\
\hline \multirow[t]{3}{*}{ Treatment response } & PR & 89 & $40.7 \pm 4.2$ & $31.9 \pm 6.2$ & 0.001 & 0.965 & 0.403 & $<0.001$ \\
\hline & SD & 43 & $27.7 \pm 3.4$ & $23.4 \pm 8.2$ & & & & \\
\hline & PD & 15 & $15.6 \pm 2.5$ & $13.0 \pm 2.9$ & & & & \\
\hline \multirow[t]{2}{*}{ LA patterns } & intense & 45 & $51.5 \pm 5.3$ & $42.3 \pm 1.8$ & $<0.001$ & $<0.001$ & & \\
\hline & moderate & 102 & $26.8 \pm 2.8$ & $19.7 \pm 2.7$ & & & & \\
\hline \multirow[t]{2}{*}{ Radiation technique } & 3D-CRT & 116 & $29.1 \pm 3.8$ & $21.2 \pm 3.0$ & 0.068 & 0.029 & 0.027 & 0.639 \\
\hline & HT & 31 & $37.4 \pm 3.4$ & $32.9 \pm 4.4$ & & & & \\
\hline TACE frequency & $1-3$ & 90 & $29.4 \pm 3.4$ & $23.1 \pm 2.4$ & 0.082 & 0.004 & 0.022 & 0.952 \\
\hline & $4-6$ & 57 & $42.1 \pm 5.1$ & $32.9 \pm 6.4$ & & & & \\
\hline
\end{tabular}

LA, Lipiodol accumulation; 3D-CRT, three-dimensional conformal radiotherapy; HT, helical tomotherapy; AFP, alpha-fetoprotein; ALP, alkaline phosphatase; PD, progressive disease; PR, partial response; SD, stable disease; SD, standard deviation; TACE, transcatheter arterial chemoembolization; $\gamma$-GT, $\gamma$-glutamyltransferase.

Patterns of lipiodol accumulation were associated with several factors, including levels of hemoglobin $(\mathrm{P}=0.022)$ and alkaline phosphatase (ALP) $\quad(\mathrm{P}=0.012)$, Child-Pugh test classification $(\mathrm{P}=0.040)$, AFP response $(\mathrm{P}=0.048)$, tumor size $(\mathrm{P}=0.026)$, and treatment response $(\mathrm{P}<0.001)$. Table 1 lists the correlation with lipiodol accumulation.

\section{Response}

Among 147 HCC patients, PR was obtained in 89 patients, SD in 43 , and $\mathrm{PD}$ in 15 , and no $\mathrm{CR}$ was observed.

To assess tumor response according to the treatment, AFP response was categorized into two groups: $>25 \%$ increase or change within $\pm 25 \%$ of AFP level after EBRT as negative response, and $>25 \%$ decrease as positive response. Positive AFP response was significantly correlated with a longer survival $(\mathrm{P}<0.001)$ (Figure 2).

\section{Pretreatment variables and results of univariate and multivariate analysis}

According to pretreatment variables, the Kaplan-Meier survival curves on univariate analysis showed that hemoglobin levels $<110 \mathrm{~g} / \mathrm{L}$, higher ALP levels, Child-Pugh liver function classification of $\mathrm{B}$, tumor diameter $\geq 10 \mathrm{~cm}$, higher AFP level, negative response of $\mathrm{AFP}$, and poor response to EBRT were related to unfavorable OS. as shown in Table 1 and Figure 2. Survival rates at 1,2, and 3 years were $86.6 \%$, $49.2 \%$, and $28.2 \%$, respectively, and median survival was 23.1 months. 

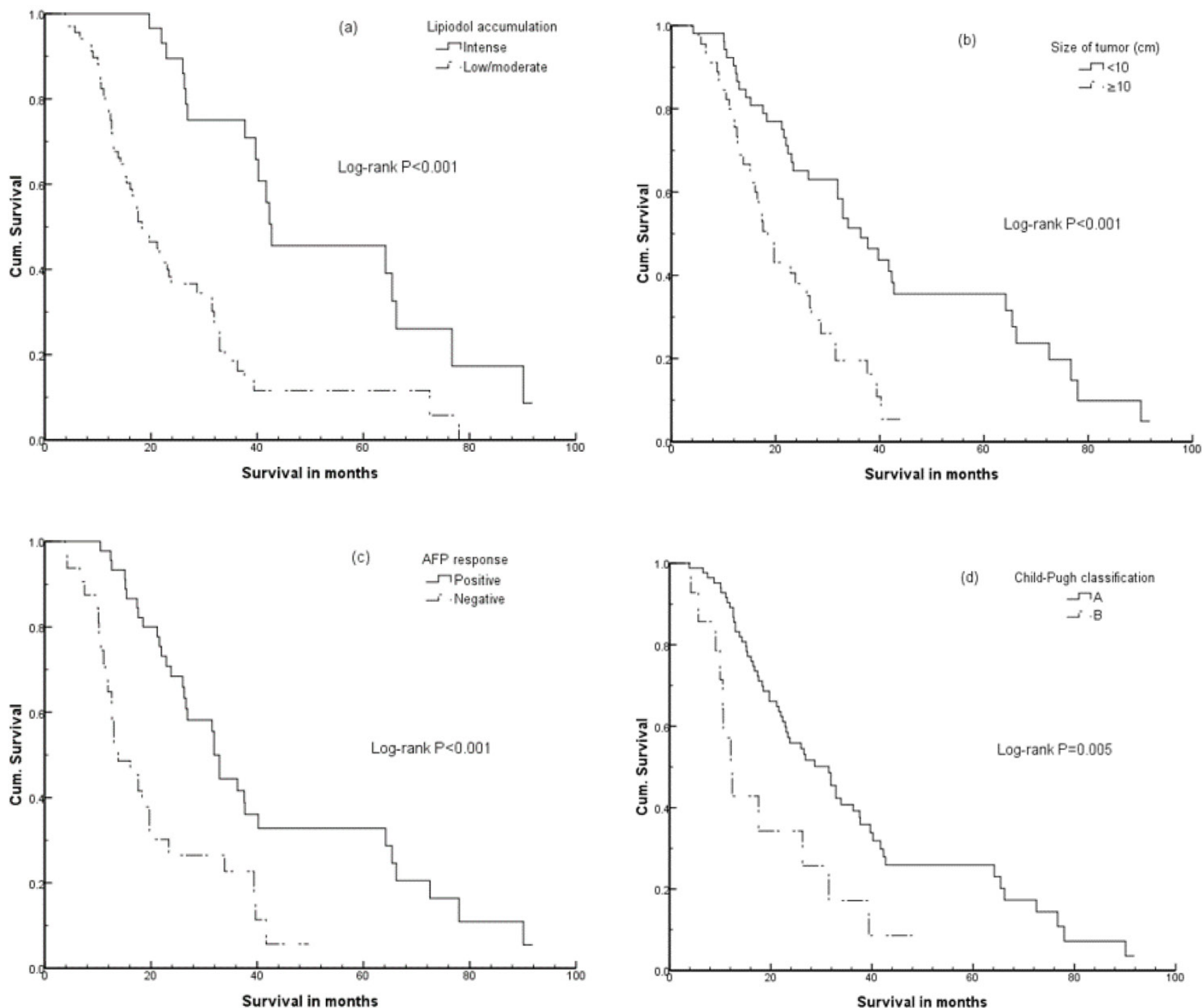

Figure 2. Cumulative (Cum) survival curves on univariate analysis according to (a) lipiodol accumulation; (b) intrahepatic tumor size; (c) AFP response; (d) the Child-Pugh test classification.

On multivariate analysis, unfavorable pretreatment predictors were associated with lower hemoglobin levels $(\mathrm{P}=0.016)$, poorer Child-Pugh liver function $(\mathrm{P}=0.037)$, lower lipiodol accumulation $(\mathrm{P}<0.001)$, negative AFP response $(\mathrm{P}<0.001)$, and using 3D-CRT technique $(\mathrm{P}=0.029)$, as shown in Table 1.

On univariate analysis, tumor size and AFP status were significantly associated with OS, but on multivariate analysis such relationships were obscure. Therefore, we performed multivariate analysis without lipiodol accumulation and found these two factors that again showed significant association with OS, as shown in Table 1 and Figure 3.

\section{Failure patterns}

Upon completion of this study, 37 patients $(25.2 \%)$ were alive, and 110 patients $(74.8 \%)$ had died. The main causes of death were hepatic failure in 79 patients $(53.7 \%)$ due to hepatic decompensation or tumor progression (or both). Lung, brain, or bone metastases were observed in 30 patients $(20.4 \%)$, with lung being the most common site in which extrahepatic metastases occurred (21 patients, 70\%); however, only 3 of the 21 patients (14.3\%) with lung metastases died from pulmonary failure. Other causes of death included gastrointestinal bleeding in 3 patients, hemolytic anemia in 2 patients, and heart attack in 1 patient.

\section{Toxicity}

Table 2 lists the sequelae of EBRT according to the Radiation Therapy Oncology Group criteria. The toxicities included increased liver enzymes after completion of EBRT (though these increases were generally less than twice the upper limit of normal), elevation of total bilirubin in patients with a history of liver cirrhosis, and decreased platelet and white blood 
cell counts (that may in part reflect portal hypertension and associated hypersplenism). Toxicities were evaluated at the first follow-up examination.

\section{Discussion}

Surgical resection is the preferred treatment for HCC patients, but most patients are not candidates due to advanced disease. TACE is the most widely used treatment for unresectable HCC, but is not curative and results in a tumor response rate $(\mathrm{CR}+$ PR) between $15 \%-55 \%$, as reported previously $[7,8]$. However, when EBRT is combined with TACE, better tumor responses, ranging from $47.4 \%-91 \%$, have been achieved $[9,10]$.

In this study, patients were diagnosed with HCC confined to the liver, but were not candidates for liver resection. Theoretically, liver transplantation is an option, as it simultaneously cures the tumor and the underlying cirrhosis, but to be eligible for transplantation, candidates must have one nodule $<5$ $\mathrm{cm}$ or up to three nodules $<3 \mathrm{~cm}$ [11]. In our study, 30 patients $(20.4 \%)$ met the standard for liver transplantation but for other reasons were unable to proceed with treatment.

We use a two-type classification to assess the degree of lipiodol accumulation instead of using the traditional four-type classification, which is based on TACE treatment alone. The two-type classification has several advantages. (1) It considers more from the prognosis and the combination of EBRT and TACE. In the traditional classification, the cumulative nonrecurrence rates and cumulative survival rates for type I were higher than those for types II-IV, but the differences among types II-IV are considerably smaller than that between type I and types II-IV [5]. (2) It is more convenient and quantifiable. Most cases can be classified visually; for ambiguous cases, we can use CT digital quantization to obtain $\mathrm{V}_{\text {lipiodol }} / \mathrm{V}_{\text {tumor }}$ and then come up with a definitive classification. (3) It is a three-dimensional assessment. Some cases present different types in different $\mathrm{CT}$ slices when using the traditional classification, which is based on two-dimensional images.

Table 2. Sequelae of external-beam radiation therapy according to RTOG criteria.

\begin{tabular}{|c|c|c|c|c|c|}
\hline \multicolumn{6}{|c|}{ RTOG Grade, n (\%) } \\
\hline & $\mathrm{n}$ & $1(\%)$ & $2(\%)$ & $3(\%)$ & $4(\%)$ \\
\hline Gastrointestinal & 147 & & & & \\
\hline Anorexia & & $44(29.9)$ & $16(10.8)$ & $6(4.1)$ & 0 \\
\hline Nausea/vomiting & & $15(10.2)$ & $3(2.0)$ & $2(1.3)$ & 0 \\
\hline Diarrhea & & $2(1.3)$ & 0 & 0 & 0 \\
\hline $\begin{array}{l}\text { Gastro/duodenal } \\
\text { ulcer }\end{array}$ & & - & $18(12.2)$ & $2(1.3)$ & 0 \\
\hline Hepatic & 147 & & & & \\
\hline Serum bilirubin & & $21(14.3)$ & $15(10.2)$ & 0 & 0 \\
\hline ALT & & 29 (19.7) & $5(3.4)$ & $2(1.3)$ & 0 \\
\hline AST & & 45 (30.6) & $16(10.9)$ & $6(4.1)$ & 0 \\
\hline ALP & & $57(38.8)$ & $8(5.4)$ & $3(2.0)$ & 0 \\
\hline Bone Marrow & 147 & & & & \\
\hline $\mathrm{Hb}$ & & $66(44.9)$ & $19(12.9)$ & 0 & 0 \\
\hline WBC & & 47 (31.3) & $15(10.2)$ & $3(2.0)$ & 0 \\
\hline Platelets & & $22(15.0)$ & $33(22.4)$ & $38(25.9)$ & 0 \\
\hline
\end{tabular}

ALP, alkaline phosphatase; ALT, alanine aminotransferase;AST, aspartate transaminase; Hb, hemoglobin; RTOG, Radiation Therapy Oncology Group; WBC, white blood cell.
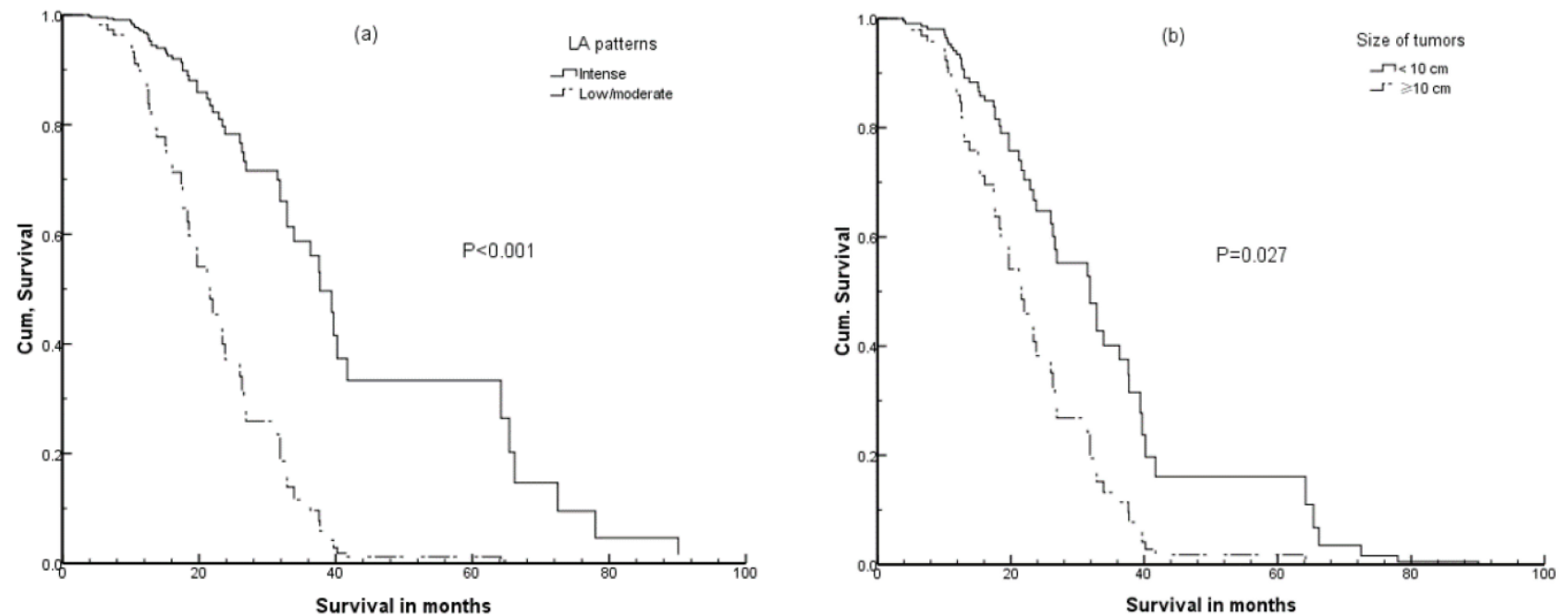

Figure 3. Cumulative (Cum) survival curves by multivariate analysis according to (a) lipiodol accumulation patterns; (b) tumor size (without lipiodol accumulation). 
Of course, our two-type classification has some disadvantages. In some large HCC tumors with dense but ring-shaped lipiodol accumulation, the center of the tumor presents as necrosis. We may classify such cases as low/moderate accumulation instead of complete/intense accumulation if $\mathrm{V}_{\text {lipiodol }} / \mathrm{V}_{\text {tumor }} \leq$ 3/4; but clinically, we consider such cases as having no residual tumor. In fact, such cases are rare (only 2 of 147 in our study), and we suggest classifying them as having complete/intense accumulation when using our classification system. New drug-eluting microspheres (beads; DEB-TACE) containing a variety of cytostatic agents are now available that are potentially more efficient than the traditional TACE. For patients receiving DEB-TACE, it is difficult to define the degree of lipiodol accumulation, although the Chinese FDA has not yet approved DEB-TACE.

Among all patients treated in this study, no case of CR was observed according to RECIST which defined CR as complete disappearance of all clinical and radiographic evidence of intrahepatic tumor. In patients who underwent combined TACE/EBRT treatment, the intrahepatic tumor did not completely disappear; in best response cases, tumors were replaced by intense lipiodol accumulation and decreased in tumor size, but these findings can only be classified as PR. Therefore, some researchers suggested that European Association for the Study of the Liver (EASL) criteria may be more precise in evaluation of therapeutic efficiency in such cases [12].

Combination of TACE with EBRT, in attempts to eliminate the residual cancer cells after TACE, results in better survival rates $[3,10]$. Therefore, we selected four factors that may be associated with tumor burden after TACE: tumor size, number of tumors, initial AFP level, and degree of lipiodol accumulation after TACE. Univariate analysis indicated a strong relationship between survival and tumor size, and also between survival and lipiodol accumulation. The local recurrence rate of small HCC $(<4 \mathrm{~cm})$ treated with subsegmental TACE has been reported to be as low as $33 \%$ after 4 years [13]. Less impressive results have been obtained for large tumors $(>5 \mathrm{~cm})$ in which complete necrosis was achieved in only $17 \%$ of cases [14]. These results indicate that larger tumor size predicts poorer survival. Multivariate analysis of our results showed that tumor size was significantly associated with poorer survival $(\mathrm{P}=0.027)$ when patterns of lipiodol accumulation were not taken into account. However, when considered, the pattern of lipiodol accumulation is a more important prognostic factor $(\mathrm{P}<0.001)$ than tumor size $(\mathrm{P}=0.177)$. This reflects the fact that tumor burden before EBRT was not assessed simply by initial tumor size but its response to TACE. As TACE obtained better local control, the residual tumor burden was less, hence, EBRT achieved a better response. In clinical practice, incomplete TACE resulted in bigger residual tumor burden, Early EBRT for HCC unresponsive to 1 or 2 cycles of TACE should be considered[15].

AFP, as most frequently used tumor marker of HCC, is a significant independent predictor of survival, patients with normal AFP levels survive longer than those with elevated levels $[16,17,18]$. Unlike AFP, ALP is a tumor marker with relatively low sensitivity and specificity, but it does show a correlation with tumor lipiodol accumulation $(\mathrm{P}=0.007)$. Multivariate analysis, disregarding lipiodol accumulation, indicated higher ALP levels are associated with poorer OS. This could be the result of two scenarios: 1) complete/intense lipiodol accumulation is seen in large tumors that produce large amounts of ALP [19]; or 2) large tumors apply pressure or obstruct the intrahepatic bile duct and impair ALP excretion that then results in elevated serum ALP levels.

Notably, the radiation therapy technique (3D-CRT vs. HT) tended to correlate with survival by univariate analysis $(\mathrm{P}=0.068)$, but was significantly related to prognostic factors by multivariate analysis $(\mathrm{P}=0.029)$. We strongly believe that the use of HT has had a major impact on the practice of radiation therapy. Patients identified to benefit most from HT are those with tumors in sites with complex anatomy, irregularly shaped tumor volumes, or tumors adjacent to radiation-sensitive normal structures, and those who receive small-volume or high-dose treatments, even in patients without good performance status and hepatic function reservation [20].

In the multivariate analysis, the frequency of TACE administration was associated with OS. However, on univariate analysis, TACE frequency did not affect survival after EBRT $(\mathrm{P}=0.817)$, and prolonged survival of patients with more TACE treatments was due to pre-EBRT factors. In this study, patients with tumor thrombus, lymph node involvement, or distant metastasis after TACE were excluded. Also those whose tumors were potentially worse in biological behavior were excluded, and such selection bias may have influenced the findings.

In conclusion, our results indicate that intense lipiodol accumulation after TACE, reflecting decrease in tumor burden, is a favorable prognostic factor for unresectable HCC patients underwent TACE and EBRT. HT is recommended as a potentially better EBRT modality than 3D-CRT in such cases.

\section{Competing Interests}

The authors state that they have no potential conflicts of interest. 


\section{References}

1. Chen WQ, Zheng RS, Baade PD, et al. Cancer statistics in China, 2015. CA Cancer J Clin. 2016;66(2):115-32.

2. Lencioni R. Loco-regional treatment of hepatocellular carcinoma. Hepatology. 2010;52:762-773

3. Zeng ZC, Tang ZY, Fan J, et al. A comparison of chemoembolization combination with and without radiotherapy for unresectable hepatocellular carcinoma. Cancer J 2004;10:307-316.

4. Bruix J, Sherman M. Management of hepatocellular carcinoma: an update. Hepatology. 2011;53(3):1020-2.

5. Nishimine $\mathrm{K}$, Uchida $\mathrm{H}$, Matsuo $\mathrm{N}$, et al. Segmental transarterial chemoembolization with Lipiodol Mixed with anticancer drugs for nonresectable hepatocellular carcinoma: follow-up CT and therapeutic results. Cancer Chemother Pharmacol 1994;33:S60-S68.

6. Therasse P, Arbuck SG, Eisenhauer EA, et al. New guidelines to evaluate the response to treatment in solid tumors. European Organization for Research and Treatment of Cancer, National Cancer Institute of the United States, National Cancer Institute of Canada. J Natl Cancer Inst. 2000;92:205-16.

7. Llovet JM, Real MI, Montana X, et al. Arterial embolization or chemoembolization versus symptomatic treatment in patients with unresectable hepatocellular carcinoma: a randomized controlled trial. Lancet 2002;259:1734-1739.

8. Lin XJ, Li QJ, Lao XM, et al. Transarterial injection of recombinant human type-5 adenovirus $\mathrm{H} 101$ in combination with transarterial chemoembolization improves overall and progressive-free survival in unresectable hepatocellular carcinoma. BMC Cancer.2015;15:707-20.

9. Zeng ZC, Tang ZY, Yang BH et al. Comparison between radioimmunotherapy and external beam radiation therapy for patients with hepatocellular carcinoma. Eur J Nucl Med 2002;291657-1668.

10. Huo YR, Eslick GD. Transcatheter arterial chemoembolization plus radiotherapy compared with chemoembolization alone for hepatocellular carcinoma: a systematic review and meta-analysis. JAMA Oncol. 2015;1(6):756-65.

11. Roayaie S, Frischer JS, Emre SH, et al. Long-term results with multimodal adjuvant therapy and liver transplantation for the treatment of hepatocellular carcinoma larger than $5 \mathrm{~cm}$. Ann Surg 2002;235:533-539.

12. Price TR, Perkins SM, Sandrasegaran K, et al. Evaluation of response after stereotactic body radiotherapy for hepatocellular carcinoma[J]. Cancer, 2012, 118(12):3191-3198.

13. Fan J, Tang $\mathrm{ZY}, \mathrm{Yu} \mathrm{Y}$, et al. Improved survival with resection after transcatheter chemoembolization for unresectable hepatocellular carcinoma. Dig Surg 1998;15:674-678.

14. Carr BI, Buch SC, Kondragunta V, et al. Tumor and liver determinants of prognosis in unresectable hepatocellular carcinoma: a case cohort study. J Gastroenterol Hepatol 2008;23:1259-66.

15. Oh D, Lim DH, Park HC, et al. Early three-dimensional conformal radiotherapy for patients with unresectable hepatocellular carcinoma after incomplete transcatheter arterial chemoembolization: a prospective evaluation of efficacy and toxicity. Am J Clin Oncol. 2010;33(4):370-5.

16. Carr BI, Buch SC, Kondragunta V. Tumor and liver determinants of prognosis in unresectable hepatocellular carcinoma: a large case cohort study. Hepatol Int. 2009 Dec 24;4(1):396-405.

17. Kitai S, Kudo M, Minami $\mathrm{Y}$, et al. A new prognostic staging system for hepatocellular carcinoma: value of the biomarker combined Japan integrated staging score. Intervirology 2008;51(S1):86-94.

18. Changchien $\mathrm{CS}$, Chen $\mathrm{CL}$, Yen $\mathrm{YH}$, et al. Analysis of 6381 hepatocellular carcinoma patients in southern Taiwan: prognostic features, treatment outcome, and survival. J Gastroenterol 2008;43:159-70.

19. Geschwind JF, Ramsey DE, Choti MA et al. Chemoembolization of hepatocellular carcinoma. Am J Clin Oncol (CCT) 2003;26:344-349.

20. Huang CM, Huang MY, Tang JY, et al. Feasibility and efficacy of helical tomotherapy in cirrhotic patients with unresectable hepatocellular carcinoma. World J Surg Oncol. 2015; 13: 201. 